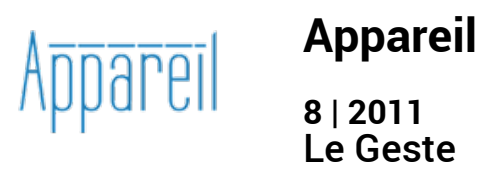

\title{
Geste, geste punitif et institution judiciaire
}

\section{Tony Ferri}

\section{(2) OpenEdition}

Journals

Édition électronique

URL : http://journals.openedition.org/appareil/1329

DOI : 10.4000/appareil.1329

ISSN : 2101-0714

Éditeur

MSH Paris Nord

\section{Référence électronique}

Tony Ferri, «Geste, geste punitif et institution judiciaire », Appareil [En ligne], 8 | 2011, mis en ligne le 07 novembre 2011, consulté le 30 juillet 2020. URL : http://journals.openedition.org/appareil/1329 ; DOI : https://doi.org/10.4000/appareil.1329

Ce document a été généré automatiquement le 30 juillet 2020.

\section{(c) (i) (2) $\Theta$}

Appareil est mis à disposition selon les termes de la Licence Creative Commons Attribution - Pas d'Utilisation Commerciale - Pas de Modification 4.0 International. 


\title{
Geste, geste punitif et institution judiciaire
}

\author{
Tony Ferri
}

Dans cet article, nous voudrions aborder la notion de geste dans ses rapports avec l'institution judiciaire. Pour ce faire, il n'est pas inutile de proposer d'abord quelques éléments conceptuels inhérents à une pensée du geste, et ce afin d'en dégager les paradoxes constitutifs. Ensuite, nous nous efforcerons de dégager la substantialité du geste punitif contemporain.

2 Posons, dans une première approximation, que la notion de geste est une notion complexe qui ne se laisse pas appréhender en dehors d'un système général de signes ou d'indices, en dehors d'un symbolisme à partir duquel, en réalité, cette notion prend sens. Un geste mérite d'être décodé pour être compris, et, lorsqu'il s'exprime à l'intérieur d'une institution qui fait écho à un ensemble de représentations ou de valeurs, il s'exécute alors selon un déroulement ou une mise en scène quasi automatique, sans besoin de réflexion préalable, un peu à la manière de la diction qui ne se réfléchit pas, mais se donne à travers une élocution aux allures d'improvisation: il est heureux que, chaque fois que l'on s'engage dans une conversation, il ne soit pas requis de faire usage d'un dictionnaire ou de demander intempestivement des arrêts, des silences qui durent et qui équivalent à des trouées dans les discours... Comme le fait de parler, le fait d'exécuter un geste n'a pas à être haché, coupé en petits morceaux, sans quoi il risquerait de perdre son sens initial, sa charge symbolique, sa dimension dialogique, sa portée communicative. Autrement dit, le paradoxe du geste tient, dans une première approche, à ceci :

- D'une part, il se donne et se comprend dans l'immédiateté de son exécution, le geste se caractérisant, dès lors, comme ne nécessitant aucune médiation ou transition. Il se donne à voir, sur le plan phénoménologique, dans l'unité ou la synthèse intransitive de son apparition. L'élève qui lève la main pour prendre la parole procède d'un rituel et d'une habitude pédagogiques propres à l'institution éducative, et cet élève qui lève la main, comme ses camarades, savent d'emblée ce que ce geste signifie. Son sens apparaît comme quelque chose de "toujours-déjà-là ", comme ce qui nous précède et nous excède, comme 
faisant déjà « monde ». En un mot, le geste ne requiert pas d'être déplié ou analysé pour être bien perçu, car il apparaît comme naturel, immédiat, sensible et foncièrement synthétique ;

- D'autre part, tout geste relève d'un mécanisme d'apprentissage, il est consécutif à un processus d'intériorisation et à une marche vers l'acquisition de connaissances. En d'autres termes, c'est un «produit» radicalement culturel qui se fabrique à l'occasion d'un mouvement analytique perçu très tôt, dès notre plus jeune âge. De là vient son lien avec la dimension institutionnelle de sa signification : la soutane des prêtres d'Église et la toge des professionnels du droit, par exemple, confèrent à ces hommes une autorité ecclésiale ou judiciaire, par cela seul que ces vêtements ont acquis, au fil du temps et des âges, le sens et la portée que l'imagination des spectateurs, une fois heurtée, veut bien leur donner. Le respect qui est attribué à ces personnes ou à ces « institutionnels ", l'autorité qui leur est conférée relèvent du rituel et du cérémonial composés d'une gestuelle et de codes particuliers, et cette autorité respectable n'appartient pas à l'ordre de la raison, mais bien plutôt à celui de l'imagination et de la croyance. Toute autorité a besoin de béquilles et d'intermédiaires, de fastes et d'apparats, d'appareils et de gestes codés pour s'instaurer et être respectée ou suivie, affirme en ce sens Pascal, au § 44 des Pensées (éd. Lafuma) :

Nos magistrats ont bien connu ce mystère, souligne-t-il. Leurs robes rouges, leurs hermines dont ils s'emmaillotent en chaffourés, les palais où ils jugent, les fleurs de lys, tout cet appareil auguste était fort nécessaire, et si les médecins n'avaient des soutanes et des mules, et que les docteurs n'eussent des bonnets carrés et des robes trop amples de quatre parties, jamais ils n'auraient dupé le monde qui ne peut résister à cette montre si authentique. S'ils avaient la véritable justice, et si les médecins avaient le vrai art de guérir, ils n'auraient que faire de bonnets carrés. La majesté de ces sciences serait assez vénérable d'elle-même, mais n'ayant que des sciences imaginaires, il faut qu'ils prennent ces vains instruments qui frappent l'imagination à laquelle ils ont affaire, et par là en effet ils s'attirent le respect.

Le geste "parle » à l'imagination, et s'institue comme pouvoir de dire, comme force d'autorité par cela seul qu'il s'incarne institutionnellement.

Dans ces conditions, il n'est pas impossible de dire du geste ce que Pascal écrit de la coutume au $\S 126$ des Pensées, à savoir qu'il est à la fois une coutume passant pour une seconde nature et une nature équivalent à une première coutume :

La coutume est une seconde nature qui détruit la première, soutient en effet Pascal dans ce fragment. Mais qu'est-ce que nature? Pourquoi la coutume n'est-elle pas naturelle? J'ai grand peur que cette nature ne soit elle-même qu'une première coutume, comme la coutume est une seconde nature.

5 En définitive, l'habitude acquise d'exécuter tel ou tel geste, le respect attaché aux gestes solennels viennent donc de leur institutionnalisation et du fait qu'ils passent pour être universels, intrinsèquement valables et raisonnables, en dépit de l'arbitraire de la survenue de leur signification et du rôle prépondérant joué dans ce registre par l'imagination: c'est dans le seul legs et l'unique réception de la signification du geste que se fonde sa légitimité apparente, sa pseudo-rationalité. Pour le dire autrement : ce n'est pas parce que le geste fait sens qu'il est institué, mais c'est, au contraire, parce qu'il est institué qu'il revêt les caractéristiques du sens et de la rationalité. Bref, le geste, en raison de sa structure éminemment culturelle, relève du factuel, de l'être, et non pas du droit ou du devoir-être. Le geste de la prière du fidèle, ou celui du bras d'honneur fait par un individu au visage empourpré, ne sont que les traductions culturelles de formes de comportement individuel et les expressions codées de sentiments particuliers (la croyance ou la colère ici), dont les modes de transmission et de communication reposent sur des mécanismes d'institutionnalisation et des 
dispositifs artificiels qui les enveloppent. En dehors des cadres institutionnels, le geste ne saurait faire sens, mais devient une gesticulation inintelligible, une succession de mouvements chaotiques, impénétrables et incompréhensibles, semblablement, dans le registre musical, à la mélodie qui se transforme en cacophonie ou en bruits insupportables et discordants par suite d'un manque de cohésion dans l'enchaînement et du désordre du jeu des notes.

6 En dépit de l'absence d'une rationalité ultime du geste, il demeure néanmoins une logique du geste: un geste se déploie, en effet, selon un commencement et une fin, selon une règle $d u$ dépliage ou du repliage, selon une succession ordonnée de mouvements précis, sans laquelle rien du geste ne pourrait être saisi par chacun et communiqué à tous. Pour reprendre la métaphore musicale, il n'est pas vain de se représenter que, de même qu'une agréable mélodie jouée en sens inverse devient un absurde tumulte, de même un geste dont les mouvements se dérouleraient à contrecourant du geste habituel (supposons le geste du coup de poing, par exemple ou, de façon plus raffinée, ceux d'un chef d'orchestre) se transformerait en l'apparition d'une agitation déréglée ou d'une folie comportementale. Par où l'on voit que sa logique est avant tout chronologique, c'est la logique du temps et de l'ordre de succession. Cette logique temporelle inhérente au geste permet de le décoder et de se l'approprier.

7 Tout geste est « au monde », pour reprendre le vocabulaire de la phénoménologie, et ne s'intègre et ne se comprend qu'à la lumière de ce legs «mondain » ou institutionnel. Si le geste se détache sur fond de monde, c'est qu'il n'a de sens qu'une fois lu et remis dans son contexte particulier d'où il naît et se développe. C'est dire, par exemple, que la position debout du parquet dans les tribunaux ou les effets de manche des avocats ne sont pas dissociables de l'institution qui tout à la fois les suppose, les implique et les façonne à son image. Décontextualisés, ces gestes perdent leur signification originelle, leur valeur heuristique. Autre exemple : démuni de sa baguette, placé en dehors du contexte de la réalisation de ses mouvements réglés, le chef d'orchestre ne se présente plus que comme un pantin désarticulé. Certes, objectera-t-on, le geste est subordonné à une intentionnalité, à un projet ou à un objectif que poursuit son exécutant. Tout n'est pas en dehors de lui, car il y a aussi un principe d'intériorité qui préside à l'efficience du geste. Mais cette intentionnalité n'est jamais rien de moins que dirigée vers un dehors qui la constitue en retour, puis reprise, récupérable et répétable par les autres : les mouvements du danseur, ceux des sportifs ne consistent pas dans une création imprévisible, mais dans le soin qu'ils apportent à les exécuter dans leur éclat originel et leur pureté primitive. La meilleure note sera attribuée au patineur qui a su reproduire, sur les plans technique et esthétique, la meilleure figure déjà connue, le plus beau saut répertorié dont les mouvements sont authentiquement décomposables en autant de moments ou de stades de développement bien identifiables et précisément acquis lors de l'entraînement. Ce qui fera la différence ici, ce n'est pas proprement le geste, mais sa technique brillante, son style, sa manière, son élégance. Autre paradoxe donc du geste : alors même qu'il est le produit de mouvements appris et répétés à satiété, il puise néanmoins sa singularité, en particulier dans le domaine de l'art au sens des beauxarts, dans le fait qu'il n'a pas son second pareil, son double, son « autre soi-même » d'un artiste à un autre, parce qu'il s'affirme selon un tempo ou un rythme différents, selon une certaine technique, selon une rapidité ou une douceur d'exécution incomparables.

8 Naturel et culturel, immédiat et transitif, partagé et unique, individuel et institutionnel dans le même temps, la notion de geste n'a pas fini de nous étonner. C'est dans le 
registre judiciaire qu'il convient maintenant de l'interroger. En effet, dans le domaine notamment des pénalités, la notion de geste prend une coloration toute particulière, puisqu'elle engage la possibilité de vivre et de mourir. En quoi, demandera-t-on, les pénalités d'aujourd'hui recouvrent-elles un aspect thanatologique en France, attendu que la peine de mort y a été abolie ? Et il semble d'autant plus opportun et pressant de se poser cette question que l'antagonisme entre les pénalités d'Ancien Régime et celles de l'époque contemporaine est criant. C'est, en effet, à un renversement de perspective sans précédent, à la faveur d'un " geste " subversif et historique, pourrait-on dire, que l'on assiste aujourd'hui, eu égard à l'histoire des pénalités, et ce consécutivement, d'une part, au repli des châtiments et des peines corporelles afflictives, infamantes et humiliantes, et, d'autre part, au recul de la peine de mort comme peine éliminatrice par excellence. Si l'on s'arrête un instant sur le principe de l'élimination du délinquant du corps social par l'exécution de la peine de mort, il est hors de doute que l'on conviendra sans difficulté que la mort pénale se devait d'observer un certain rituel et un ensemble de pratiques habituelles pour être mener à son terme. Sous l'Ancien Régime, les dynasties des bourreaux, ces familles où on naissait et mourait bourreau ou «Brisegarrot ", possédaient l'incroyable "privilège » de tuer publiquement et légalement ceux que le roi et les seigneurs désignaient à la vindicte publique et populaire, ceux-là même qui avaient eu l'audace et l'inconscience de transgresser les lois du royaume. Les bourreaux, vêtus de leur tenue ordinaire de cérémonie de mise à mort, à savoir une casaque, marquée par un signe distinctif signalant leur office odieux, de couleur rouge sang de bœuf et des bottes, et ses aides portant une casaque noire et des sabots, étaient tenus de suivre un protocole précis dans les gestes qu'ils avaient à effectuer, sous peine de voir la foule se retourner contre eux. Les gens des villes ou des villages, affluant et venus assister aux exécutions, désiraient voir, sur la scène où se déroulait la cérémonie de la mise à mort notamment par décapitation (réservée grosso modo à la noblesse, car la hache faisait honneur), par pendaison (destinée généralement à la plèbe, car la corde faisait honte), ou, pire, par l'épreuve de la roue ou par écartèlement pour les régicides, un spectacle très laid, mais pas radicalement effrayant. Les bourreaux le savaient bien. Le protocole gestuel de la pendaison, en tant qu'elle constituait la modalité d'exécution de peine la plus répandue, consistait, selon l'étude instructive et bien documentée de J. Delarue, en ceci :

À Paris, le pendu a presque toujours la tête luxée, parce que la corde, placée sous la mâchoire et l'os occipital, fait une contre-extension et que (horrible détail) l'exécuteur monte sur les mains liées du patient, qui lui servent comme d'étrier. Il agite violemment le corps, de haut en bas, en donnant des secousses, 'puis il fait faire au tronc des mouvements demi-circulaires, alternatifs et très prompts, d'où suit une luxation de la première vertèbre'. À Lyon, par contre, l'exécuteur place le nœud coulant sur la nuque, la corde étant munie d'un second nœud fixe, placé en avant, qui empêche la corde de glisser sous le menton. L'exécuteur (détail plus horrible encore) monte sur la tête du 'patient' et tire en avant. Le nœud pèse alors sur le larynx ou la trachée artère, et provoque lacération et déchirure ${ }^{1}$.

Il en va de même de l'utilisation, plusieurs décennies plus tard, de la fameuse « bascule à Charlot ", c'est-à-dire de la guillotine, appelée ainsi dans les milieux populaires et délinquants, par référence au fait que le prénom commun et fort banal des exécuteurs était celui de Charles, et cela de génération en génération. À la suite de la fabrication de cet appareil à bascule, les gestes de mise à mort furent commandés davantage par la cadence des exécutions, la logique du rendement et l'efficacité du résultat que par le seul spectacle lui-même destiné initialement à dissuader du crime, à marquer les 
esprits et à montrer l'éclat réel du pouvoir. Durant la période de la Terreur jacobine, la guillotine ne connut pas grand repos:

La tourmente révolutionnaire avait fait de la guillotine l'instrument des exécutions au niveau industriel, souligne, plus loin, J. Delarue. Dans ces séries de quarante à cinquante condamnés, le rythme extraordinaire auquel parvenaient le bourreau et ses aides, entraînés il est vrai par une pratique bi-quotidienne, permettait d'exécuter chaque condamné en à peine plus de deux minutes. Aucun autre moyen n'aurait permis une telle cadence. Nous connaissons la technique qui permit aux exécuteurs ce sinistre record. Normalement, le condamné est amené face à la planche dite 'bascule', levée. On le lie à cette planche à l'aide de deux fortes courroies situées à mi-hauteur du dos et à hauteur des genoux. Puis, les aides abattent la bascule, et le condamné se trouve couché sur le ventre, la tête dans le cercle de la lunette ouverte. Le chef ferme alors la lunette, et déclenche la chute du couperet. Pour les séries révolutionnaires, la bascule demeurait abaissée. L'exécuteur avait toujours avec lui sur l'échafaud ses quatre aides, à Paris tout au moins. Quand le condamné arrivait sur la plate-forme, l'un des aides lui saisissait le bras droit, un autre le bras gauche, un troisième saisissait les jambes. Tous trois le soulevaient et le jetaient sur la bascule, où il se trouvait couché sur le ventre. On ne prenait pas la peine de le sangler, les trois aides continuaient à le maintenir. Le quatrième, placé face à la lunette, ajustait la tête dans le cercle de celle-ci, qui se fermait instantanément, et le couperet s'abattait presque en même temps. Pendant que le quatrième aide remontait le couperet, les trois autres jetaient le corps directement dans un tombereau rangé à côté de l'échafaud, puis se retournaient et saisissaient le suivant qui arrivait en haut de l'escalier. Les têtes tombaient dans un grand sac contenant de la sciure, accroché devant la lunette ${ }^{2}$.

10 Au-delà des scènes d'horreur que ces exécutions ne manquaient pas de faire voir, le motif bien connu de ces mises à mort reposait sur la nécessité d'éliminer du corps social le délinquant, en tant qu'il transgressait les lois, troublait l'ordre établi et portait atteinte à la dignité même du roi et à la grandeur des seigneurs. Il s'agissait, par là, de faire disparaître définitivement l'Outlaw, de le propulser hors du champ communautaire, bref de le rendre invisible au moyen d'une technologie éprouvée et, quant à elle, bien visible, à savoir l'échafaud. Pour le dire autrement: la mort du supplicié consistait à le rendre totalement invisible et indiscernable (il s'agissait même, dans certains cas, d'effacer toute trace de son existence, en chassant sa famille, en brûlant sa maison, etc.) par des moyens qui étaient, eux, macroscopiques, très visibles et excessivement manifestes ou évidents.

Le renversement de perspective, propre à notre présent, tient au fait qu'il s'agit dorénavant de soustraire le condamné à la visibilité de la sanction, de rendre invisibles et discrets les moyens d'exécution mis en œuvre, au profit du maintien de sa visibilité sociale en tant que délinquant. C'est bel et bien un renversement complet du sens de la peine qui est ici mis à jour par les pénalités nouvelles. Et lorsqu'il est évoqué ici ces pénalités nouvelles, il ne saurait être question de faire référence à l'emprisonnement qui - en tant que peine de relégation, jadis dans des cachots obscurs placés dans les sous-sols des bâtiments ou dans des "enfermeries ", aujourd'hui dans des cellules de prisons pour peine-, consacra le principe de l'élimination du délinquant de la collectivité (invisibilité du condamné), selon des moyens macroscopiques et massifs (l'établissement pénitentiaire actuel de Fleury-Mérogis, en tant qu'édifice extraordinaire de béton incontestablement perceptible, offre une remarquable illustration des moyens mis en œuvre pour punir). Ce dont il s'agit aujourd'hui, pour le législateur, c'est de télescoper les gestes classiques et punitifs d'exclusion comme l'emprisonnement par des gestes punitifs et novateurs d'inclusion formés et intronisés 
par la surveillance électronique. L'individu placé sous surveillance électronique (le PSE) intègre, rappelons-le, un dispositif de contrôle qui n'est pas apparent, en portant notamment à sa cheville un bracelet électronique dissimulé sous sa chaussette. Ce dispositif a pour objectif de permettre la réintégration du condamné dans la communauté, de le rendre mobile parmi ses semblables, de le réhabiliter par le travail, de maintenir les liens familiaux par la fréquentation facilitée de la famille, bref de le remobiliser socialement. Les moyens mis en œuvre sont, quant à eux, indécelables, en tout cas difficilement repérables, car la surveillance s'exerce de manière diffuse, partout et nulle part dans le même temps, et de façon permanente en ce qu'elle ne prévient pas de son regard. Le geste punitif d'aujourd'hui est celui du regard asymétrique et non réciproque, celui de l'œil scrutateur ou inquisiteur. Au geste d'une punition au sédentarisme ou à l'immobilisme (la prison) se substitue peu à peu celui d'une punition au nomadisme et à la mobilité (le Placement sous surveillance électronique). Autre aspect de ce renversement de perspective: tandis que le vagabondage fut gravement réprimé sous l'Ancien Régime, car le vagabond était perçu comme appartenant à la classe dangereuse, par cela seul qu'il passait entre les mailles du filet du contrôle social par le travail et le logement, et parce qu'il échappait à toute topographie identifiable, fuyant souvent d'abord son village, errant ensuite de ville en ville, il était donc regardé comme ce personnage improductif et vivant de petites rapines, et considéré comme le menu fretin des gens du peuple. Ce que permet, en revanche, le Placement sous surveillance électronique, c'est le retour de la déambulation et de l'errance comme principe compatible avec les intérêts de la société, et ce retour s'explique justement par l'efficacité des dispositifs de contrôle d'aujourd'hui et la technologie électronique. M. Foucault l'avait bien perçu: la vadrouille des condamnés est moins coûteuse économiquement au regard des efforts qui doivent être déployés pour construire des prisons, recruter des surveillants et administrer les services, et plus efficace pénalement, à la condition expresse toutefois qu'elle suscite la participation des condamnés à leur propre contrôle et des comportements d'auto-observation. La surveillance électronique généralisée est un avatar du panoptique de Bentham, parce qu'elle place l'individu surveillé dans des réflexes et des conditionnements comportementaux impliquant l'acceptation de l'enrégimentement, l'auto-régularisation, l'auto-normalisation, la fabrication de son propre moule où il doit se couler automatiquement. Le panoptique de Bentham, cette « ruche faite de verre », n'est plus une gageure ou une légende, mais une réalité pénale permise par l'avènement de la technologie électronique. Si l'idée du panoptique est à relier aux découvertes et au développement des connaissances en optique, à l'époque moderne, elle trouve son ultime fécondité dans l'achèvement par la technologie électronique d'une surveillance à distance, sans visage ni nom, anonyme, diffuse, impalpable, productive et participative. Dès lors, dans cette configuration générale des pénalités nouvelles, il appert qu'il n'est plus besoin de recourir à des peines d'élimination expéditives et ostentatoires, mais à des sanctions d'intégration numériques et discrètes. Le geste pénal ne consiste plus à faire souffrir le corps par l'exécution des pires châtiments ou à l'éliminer, mais à maintenir en vie le condamné par des mécanismes d'emprise sur ses états psychiques et sur son intimité, et par des techniques d'influence, des méthodes d'auto-discipline favorisant, chez lui, le renoncement volontaire à jouir d'une autonomie réelle.

Une des conséquences souvent tue de ce renversement du geste punitif est l'éviction progressive du savoir-pouvoir des éducateurs. En effet, alors que les prisons requièrent 
l'organisation de rencontres entre les éducateurs et les délinquants en vue de préparer la sortie et la réintégration de ces derniers, alors qu'elle suppose l'articulation du savoir de ces professionnels de la réinsertion sur la conduite des infracteurs susceptibles de passer à l'acte ou soupçonnables de récidive, alors que, en dernière analyse, le savoir particulier des éducateurs de prison renforçait la légitimité et l'efficacité du pouvoir de l'État et consolidait, dans le même temps, la place et le rôle du travail éducatif dans les milieux clos des établissements pénitentiaires, l'avènement de la supervision électronique a provoqué un relâchement décisif du statut des éducateurs dont la mission était notamment destinée à élever la conduite des délinquants au rang des normes sociales acceptables et au niveau de cette posture requise par l'ordre social lui-même. Ce n'est pas un hasard si, dans ce contexte d'appauvrissement des liens humains, l'appellation même d'éducateur de prison n'existe plus, et qu'elle a été supplantée par une autre, en 1993, à savoir par celle de conseiller d'insertion et de probation, qui, au demeurant, ne veut plus rien dire. S'il est louable pour certains d'abandonner le modèle de la prison comme institution de la mort (cf. M. Foucault) et comme établissement "fossile ", il est décevant pour d'autres, sans pour cela y voir le basculement dans des logiques " maternelles » ou "paternalistes", de renoncer à tout projet de réinsertion et à tout principe d'aide, ainsi que de rompre avec l'intention bienveillante et attentionnée de redynamiser socialement ceux qui ont besoin d'être soutenus, reconnus, reclassés.

13 Au final, la structure même du geste punitif d'aujourd'hui tend à se laisser relayer par des logiques purement mécaniques, procédurales et numériques. Il ne s'agit plus seulement pour les surveillants de se familiariser avec les techniques du contact, comme la palpation ou la fouille à corps, il n'est plus seulement question pour les conseillers d'insertion et de probation de maîtriser les techniques d'entretien et de prise en charge, mais il s'agit, pour l'institution péno-pénitentiaire, de dompter les mécanismes de supervision à distance et les procédures d'exécution de peine relatives aux diverses mesures restrictives de liberté promises à un riche avenir. L'ingéniosité de tels dispositifs de contrôle électroniques vient de ce qu'elle ne laisse pas de place, ne serait-ce que dans un petit intervalle de temps, au sentiment de l'autonomie réelle, à la pertinence du dialogue et à la possibilité de la contestation. En écartant le modèle pastoral de la réhabilitation, fondé sur l'accompagnement social des « déjà » anciens éducateurs, le bracelet électronique exige une exécution des peines sans condition, confie la spécialisation de la "réformation » à un système électronique fonctionnant seul (car il s'agit bien de prévenir la récidive, et donc d'impulser les conditions d'un changement dans le cœur et l'âme des condamnés) et fait disparaitre tout espoir d'une intériorisation dynamique, créatrice, porteuse de transformation, empreinte de liberté et respectueuse de la personne, en raison de la mécanicité et de l'anonymat du suivi. À l'intérieur de ce geste punitif intrusif que représente la surveillance électronique, il n'y a plus place pour cet interstice vital, pour cet intervalle de temps microscopique et pourtant précieux, pour ce trou marginal, où se concentrent ces causes infinitésimales, qui concourent à la genèse d'une conduite authentiquement humaine, par cela seul que cette conduite n'est pas traquée jusque chez elle et qu'elle n'a pas à craindre la sanction mécanique du faux pas ou de l'écart directionnel. 


\section{NOTES}

1. Jacques Delarue, Le Métier de bourreau, Paris, Fayard, 1979, p. 144.

2. Ibid., p. 179.

\section{RÉSUMÉS}

À l'aube du nouveau millénaire, se sont développées, notamment en France, à la suite d'expériences menées en particulier aux États-Unis, au Canada et en Suède, de nouvelles technologies du pouvoir punitif. Ces technologies, qui se traduisent par des dispositifs de contrôle inédits, à savoir précisément la surveillance électronique, apparaissent, de par la place importante qu'elles occupent dans le champ des pénalités et de par l'investissement intensif des pouvoirs publics qu'elles commandent aujourd'hui, comme ne devant désormais rencontrer aucun frein. Dès lors, c'est de tenter de circonscrire ici le principe organisationnel et fonctionnel inauguré par ces nouvelles technologies d'enfermement, puissamment dopées à l'électronique, que se propose cet article, et ce à travers la mise à plat d'éléments de réflexion sur la structure d'un des gestes punitifs contemporains des plus audacieux, des plus " renversants ", et des plus malheureux sur le plan de la qualité et du sens de la prise en charge des condamnés.

\section{INDEX}

Mots-clés : bracelet, contrôle, dispositif, pénalité, surveillance électronique, technologie

Personnes citées : Foucault (Michel)

\section{AUTEUR}

\section{TONY FERRI}

Conseiller d'insertion et de probation du ministère de la Justice. Doctorant en philosophie à

l'université Paris 8, tony.philosophe@yahoo.fr. 